\title{
Interpolation Using Cubic Bèzier Triangular Patches
}

\author{
Samsul Ariffin Abdul Karim, Azizan Saaban*, Mohammad Khatim Hasan ${ }^{\$}$, Jumat Sulaiman ${ }^{+}$, \\ Ishak Hashim ${ }^{\#}$ \\ ${ }^{\#}$ Fundamental and Applied Sciences Department and Centre for Smart Grid Energy Research (CSMER), Institute of Autonomous System \\ Universiti Teknologi PETRONAS, Bandar Seri Iskandar, 32610 Seri Iskandar, Perak DR, Malaysia \\ E-mail:samsul_ariffin@utp.edu.my
}

*School of Quantitative Sciences, UUMCAS, Universiti Utara Malaysia, 06010 Sintok, Kedah DA, Malaysia E-mail:azizan.s@uum.edu.my

\$Center for Artificial Intelligence and Technology, Faculty of Information Science and Technology, Universiti Kebangsaan Malaysia, 43600 UKM Bangi, Selangor, Malaysia.

E-mail:mkh@ukm.edu.my

${ }^{+}$Programme of Mathematic with Economy, School of Science and Technology, Universiti Malaysia Sabah, Locked bag 2073, 88999 Kota Kinabalu, Sabah

E-mail: jumat@ums.edu.my

\begin{abstract}
Spatial interpolation is a method that can be used to estimate the unknown value (or parameter) at certain time or location. Besides of that, shape preserving interpolation especially positivity preserving interpolation play an important role for data visualization where the resulting interpolating surface must be positive everywhere. In this study, we use a triangulation based method consists of positivity preserving cubic Bézier triangular patches to estimate the unknown value of rainfall amount at spatial localization. This scheme is useful for spatial data interpolation compared to the mesh free methods which are required the optimization technique in order to ensure the positivity of basis functions. The data is triangulated by using Delaunay's triangulation algorithm and from there the triangular surfaces are constructed piecewisely comprising three local schemes that are blended together via convex combination to form a scattered interpolating surface. To preserve the positivity of the surface, the appropriate conditions for the Bézier ordinates are implemented. Validation of the propose schemes are conducted by using wellknown test function used in previous researches. Then, the best the scheme is selected to visualize a rainfall data distribution based on secondary data collected at various meteorology stations in Malaysia obtain from Malaysian Meteorology Department. Finally, we estimate the unknown rainfall amount at certain locations that lies inside the convex hull formed by all given stations. The finding shows that positivity preserving cubic triangular patches is able to preserve the positivity of rainfall distribution surface and has a significant application in estimating the rainfall amount at unallocated meteorology stations in Malaysia.
\end{abstract}

Keywords - spatial interpolation; cubic Bezier; positivity preserving; rainfall.

\section{INTRODUCTION}

Spatial interpolation is important in geophysical data processing and prediction since this method capable to estimate the unknown value of the geophysical event at certain time or location Besides that, the interpolation also has roles in planning, risk assessment and environmental management. There are many methods that can be used as a tool to estimate the unknown value using every known points which is the global interpolation or using sample of known points known as local interpolation. One of the earliest global interpolation method is Shepard's method also known as Inverse Weighted Distance (IDW) [1]. Later many researchers have utilized different types of bases functions such as meshfree methods i.e. RBF and thin plate spline, bi-cubic spline interpolation and nearest neighbor interpolation. Lodha and Franke [1], Franke and Nielson [2], Franke [3] and Franke and Nielson [4] give a good survey on the scattered data techniques for surfaces interpolation

An alternative strategy is by using local interpolation method based on the Bézier triangular patches in a form of piecewise locally constructed surfaces. Triangular elements have been use in many researches, especially, to increase the smoothness of images, such as research in [5] and [6]. Li et al. [5] use texture synthesis while Miroslav et al. [6] apply 
Hermite interpolation. Fig. 1 shows an example of triangular applications to Batman face meshes [18] while Fig. 2 shows a surface that discretized using triangular patches with high resolution (bottom) and low resolution (top). From Fig. 2, it is clear that using higher resolution of patches, the constucted surface is more smooth compared to the low resolution of triangular patches [19]. Beside of the triangular patches, the rectangular patches also widely used in many applications. Fig. 3 [20] shows the comparison of the surface reconstruction using triangular and rectangular patches. One of the advantage using the Bézier triangular is that we are able to reconstruct the piecewise surface with some degree of smoothness attained and the interpolating surface can be controlled locally [7]-[9].

Besides that, shape preserving interpolation and approximation for data visualization is important in real world problems. For instance, rainfall distribution is always positive value, then the resulting interpolating surface must also be positive everywhere. This can be achieved by implementing the appropriate positivity condition that will produce positive interpolant to the positive data. Piah et al. [7] studied the positivity preserving scattered data interpolation by using cubic Bézier triangular. They derived the sufficient condition for positivity on the Bézier ordinates. Their condition is more relaxed compare with the sufficient condition in Chan and Ong [10]. Saaban et al. [8] utilized the quintic Bézier triangular to construct positive scattered data interpolation. Goodman and Said [11] constructed the $C^{1}$ continuity triangle interpolant using convex combination scheme consist of three local schemes. Their work is difference compare to Foley and Optiz [12], eventhough both studies developed a $C^{1}$ cubic triangular convex combination scheme. This approach is extend by Chang and Said [13] using $C^{2}$ continuity based on quintic triangular spatches which requires the partial derivative values up to the order two. Besides the use of Bézier triangular, many authors construct the positive scattered data interpolation by using meshfree methods such as Gaussian, Radial Bass Functions (RBF) etc. For instance, Brodlie et al. [14] use the Shepard quadratic basis function to construct the positive interpolant for positive data. Their scheme requires the optimization method in order to produce the positive interpolant everywhere. Skala [15] apply RBF Interpolation by considering Compactly Supported RBFs (CSRBF) of large data sets.

The aim of this study is to construct the piecewise triangular surface for scattered data by using cubic Bézier triangular patches involving three local schemes that are blended together via convex combination. We test the scheme to estimate the unknown value for rainfall volume at certain location that lies inside the convex hull formed by the given scattered data points. This scheme is useful for spatial data interpolation and does not require the optimization technique as appear in the work of Brodlie et al. [14].

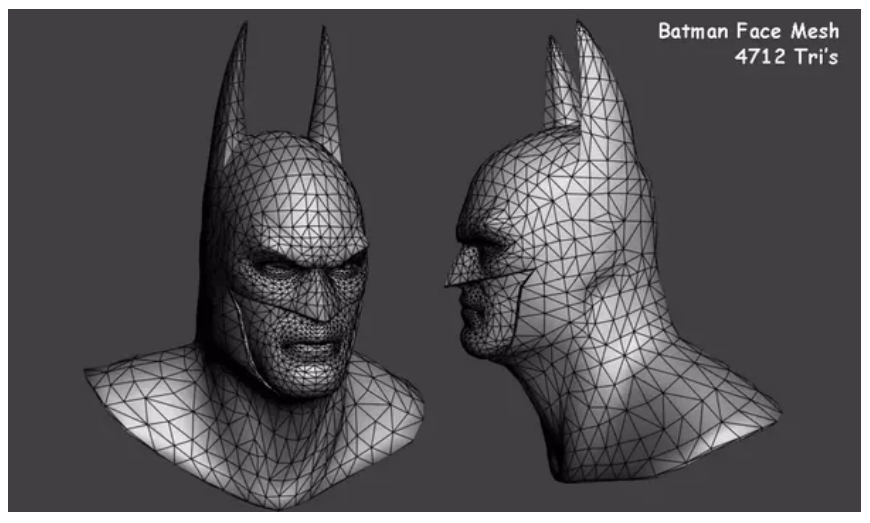

Fig. 1 Example of triangular patches application.

\section{MATERIAL AND METHOD}

The cubic Bézier triangular is given as, (Farin $[16,17])$

$$
P(r, s, t)=\sum_{i+j+k=3} c_{i j k} r^{i} s^{j} t^{k}, \mathrm{r}+s+t=1
$$

Equation (1) also can be represented by

$$
\begin{aligned}
P(r, s, t)= & r^{3} c_{300}+s^{3} c_{030}+t^{3} c_{003}+3 r^{2} s c_{210} \\
+3 r^{2} t c_{201} & +3 s^{2} r_{120}+3 s^{2} t c_{021}+3 t^{2} r c_{102} \\
& +3 t^{2} s c_{012}+6 r s t c_{111}
\end{aligned}
$$
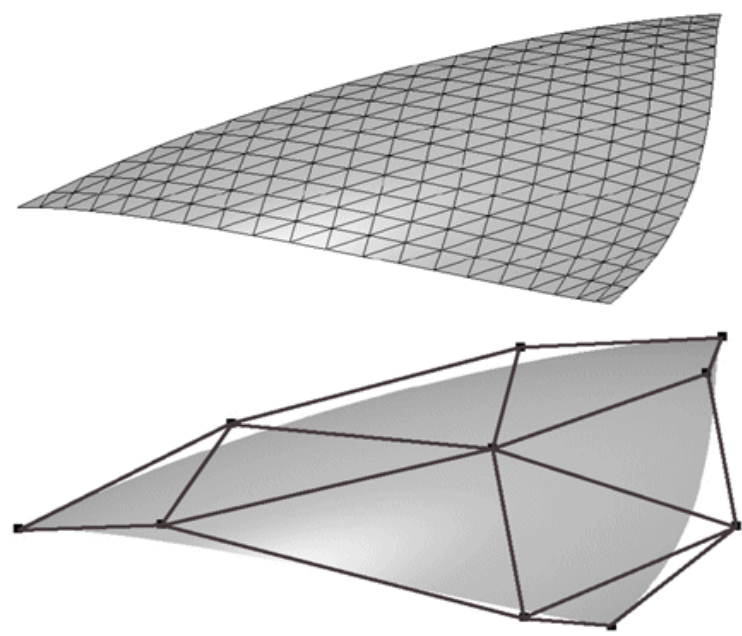

Fig. 2 Discretization of a surface using triangular patches.

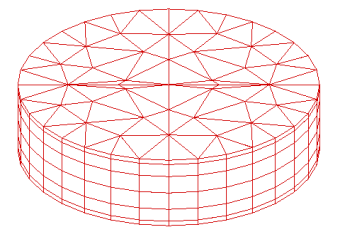

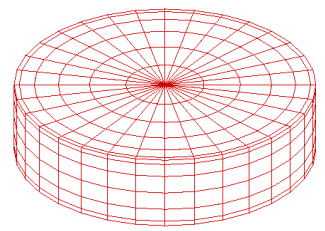

b
Fig. 3 Discretization of a surface using (a) triangular patches and (b) rectangular patches. 
The de Casteljau algorithm can be used to construct the cubic Bézier triangular patches. With 10 control points, the cubic Bézier triangular patch will lies inside the convex hull forming by all 10 control points.

Fig. 4 shows the Bézier ordinates for the cubic Bézier triangular on one patch and Fig. 5 shows the cubic Bézier triangular bases. To derive the positivity sufficient condition of the cubic Bézier triangular defined in Eq. (2), the following assumption has been made:

\section{Assumption 1:}

The Vertices Bézier ordinates are always positive i.e. $c_{300}>0, c_{030}>0$ and $c_{003}>0$ respectively.

There are three different positivity sufficient conditions for the Eq. (2). The interested reader can refer to Chan and Ong [10], Piah et al. [7] and Saaban [9] for cubic cases and Saaban et al. [13] for quintic case.

\section{Theorem 1 [2].}

Let the cubic Bézier triangular patch $P(u, v, w)$ in (1) with $u$, $v, w \geq 0, u+v+w=1$, where $c_{300}=\alpha \ell, c_{030}=\beta \ell, c_{003}=\ell, \ell$

$$
>\quad \text { and } \quad \alpha \geq \beta \geq 1 \text {. }
$$

If $c_{210}, c_{201}, c_{120}, c_{021}, c_{012}, c_{102}, c_{111} \geq-\frac{\ell}{3 a}$, where $a$ is in $(1,8 / 3]$ is a unique solution to the following nonlinear equation

$$
\begin{aligned}
& 16-8 \alpha+\left(72 \alpha-27 \alpha^{2}\right) a+54 \alpha^{2} a^{2}-27 \alpha^{2} a^{3}=0, \\
& P(u, v, w) \geq 0, \forall u, v, w \geq 0, u+v+w=1 .
\end{aligned}
$$

then

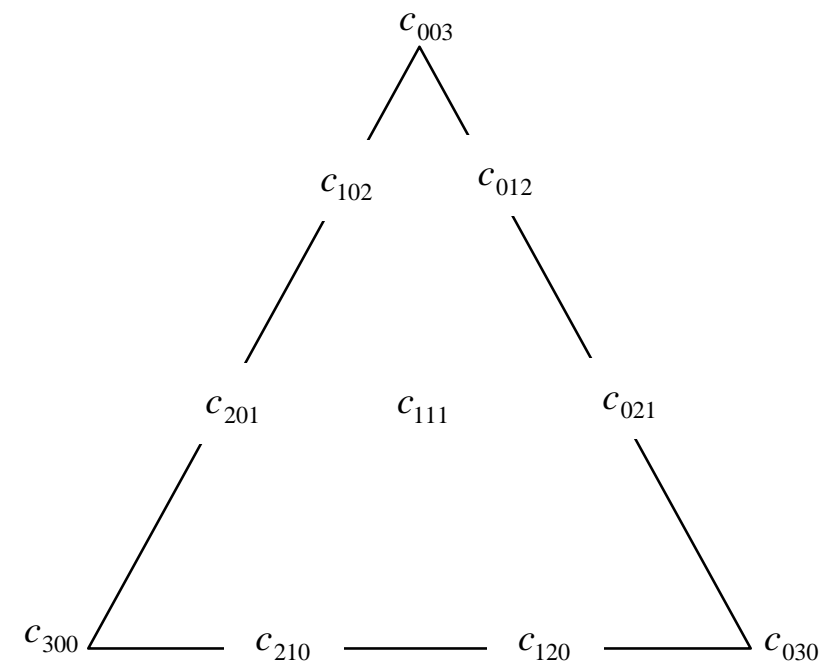

Fig. 4. Cubic Bézier points (control points)

\section{Theorem 2 [7].}

Consider the cubic Bézier triangular patch $P(u, v, w)$ in (1) with $c_{300}=A, c_{030}=B, c_{003}=C, A, B, C>0$. If $c_{210}, c_{201}, c_{120}, c_{021}, c_{012}, c_{102}, c_{111} \geq-\frac{1}{s_{0}}, s_{0}$ is the unique solution of the $G(s)=1$ with $G(s)=\frac{1}{\sqrt{A s+1}}+\frac{1}{\sqrt{B s+1}}+\frac{1}{\sqrt{C s+1}} \quad$ then $P(u, v, w) \geq 0 \forall u, v, w \geq 0, u+v+w=1$.

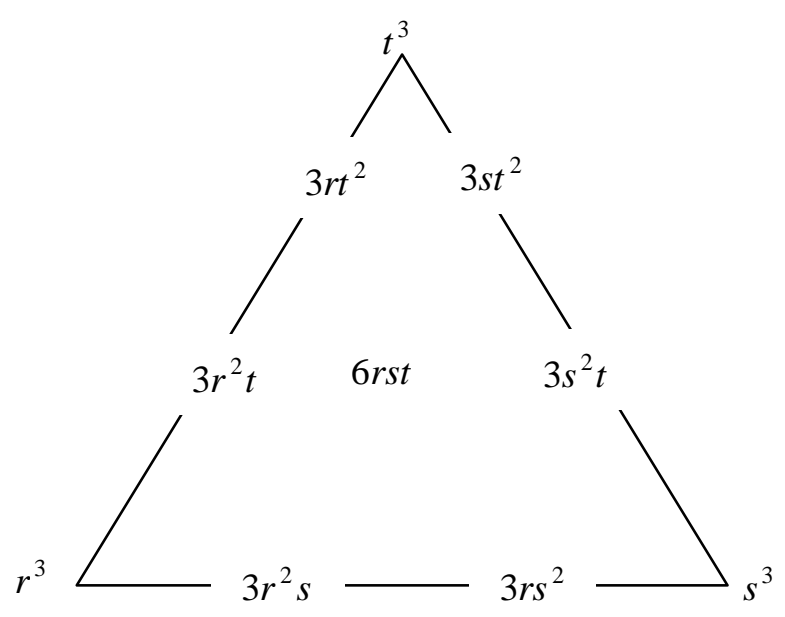

Fig. 5. Cubic Bézier triangular bases

Theorem 3 ([9]).

Consider the cubic Bézier triangular patch $P(u, v, w)$ in (1) with $\mathrm{c}_{300}=A, c_{030}=B, c_{003}=C$ where $A, B, C>0$. If $c_{021}$, $c_{012} \geq-y_{1}{ }^{0}, c_{201}, c_{102} \geq-y_{2}{ }^{0}, c_{210}, c_{120} \geq-y_{3}{ }^{0}$ and $c_{111} \geq-m_{0}, y_{1}$ ${ }^{0}, m_{0}>0, i=1,2,3$ such that $y_{1}^{0}$ is the unique solution of $3 y_{1}{ }^{4}+4(B+C) y_{1}{ }^{3}+6 B C y_{1}{ }^{2}-B^{2} C^{2}=0$ in $(0, B+C), y_{2}{ }^{0}$ is the unique solution of $3 y_{2}{ }^{4}+4(C+A) y_{2}{ }^{3}+6 C A y_{2}{ }^{2}-C^{2} A^{2}=0$ in $(0, C+A)$, where $y_{3}{ }^{0}$ is a unique solution of the nonlinear equation $3 y_{3}{ }^{4}+4(A+B) y_{3}{ }^{3}+6 A B y_{3}{ }^{2}-A^{2} B^{2}=0$ in $(0$, $A+B)$ and

$$
m_{0}=\frac{\left(A+y_{0}\right)^{\frac{1}{3}}\left(B+y_{0}\right)^{\frac{1}{3}}\left(C+y_{0}\right)^{\frac{1}{3}}}{2}+\frac{\left(6-a^{3}\right) y_{0}}{6}
$$
with $\quad y_{0}=\min \left(y_{1}{ }^{0}, y_{2}{ }^{0}, y_{3}{ }^{0}\right)$ and $a$ is given by $\frac{\left(\left(B+y_{0}\right)\left(C+y_{0}\right)\right)^{\frac{1}{3}}+\left(\left(A+y_{0}\right)\left(C+y_{0}\right)\right)^{\frac{1}{3}}+\left(\left(A+y_{0}\right)\left(B+y_{0}\right)\right)^{\frac{1}{3}}}{\left(\left(A+y_{0}\right)\left(B+y_{0}\right)\left(C+y_{0}\right)\right)^{\frac{2}{9}}} \mathrm{t}$ hen $P(u, v, w) \geq 0$ for all $u, v, w \geq 0, u+v+w=1$.

\section{RESULTS AND DISCUSSION}

We test the positivity preserving of above the three schemes by using the 36 data points obtain from the test function $f(x, y)$ as listed in Table I. The function is given by

$$
f(x, y)=\left\{\begin{array}{cc}
1.0 & (y-x) \geq 0.5 \\
2(y-x) & 0 .(y-x) \leq 0.5 \\
\frac{\cos (A)+1}{2} & (x-1.5)^{2}+(y-0.5)^{2} \leq 1 / 16 \\
0 & {[0,2] \times[0,1]}
\end{array}\right.
$$

where

$$
A=4 \pi \sqrt{(x-1.5)^{2}+(y-0.5)^{2}}
$$


Fig. 6 show the Delaunay triangulation for the 36 data points (nodes). Fig. 7 shows the result obtained by using the scheme of Chan and Ong [10] with (a) without positivity preserving and (b) with positivity preserving. Even though Chan and Ong include the positivity preserving element, some of the simulated data still producing negative values. Fig. 8 shows result approximated by scheme proposed by Piah et al. [7] where (a) without positivity preserving and (b) with positivity preserving property. Piah et al. [7] succeed to produce non-negative simulated values. This scheme is able to to improve the performance of [10].

The positivity preserving by using the scheme of Saaban [9] is shown in Fig. 9(b) and the interpolating surface without positivity preserving i.e. not satisfy the sufficient condition for positivity preserving is given in Figure 9(a).

From Figs. 7-9, it is can clearly be seen that, Chan and Ong [10] scheme give some negative values even though the cubic Bézier interpolant satisfies the sufficient condition for positivity given in Theorem 1 . In contrasts to Chan and Ong [10], the schemes presented by Saaban et al. [8] and Saaban [9] preserves the positivity of the data and give more relaxed condition compare with the conditions given in Chan and Ong [10]. Saaban [9] also conjectured that his scheme is better that Chan and Ong [10] and Piah et al. [7] in terms of the value of coefficient of determination $\mathrm{R}^{2}$.

Next we test the scheme of [9] for positivity preserving interpolation of real data i.e. rainfall data collected at various station in Malaysia as in Table II. Fig. 9 shows the linear interpolant of the rainfall data. Fig. 10 shows the surface of rainfall distribution in Malaysia. Fig. 11(a) shows the surface interpolation without positivity preserving while Fig. 11(b) shows that the interpolating surface with shape preserving interpolation is positive everywhere by imposing condition given in Theorem 3 .

To achieve this, we use MATLAB programming that need the user to input the longitude and latitude value for the desired location that lie inside the convex hull formed by the rainfall stations in Peninsular Malaysia. Table II summarized the estimated value for rainfall volume in $\mathrm{mm}$ at eight (8) selected locations in Peninsular Malaysia.

Based on graphical illustrations, we can see that the positivity preserving using the scheme of Saaban [9] and Piah et al. [7] give good results compared with the scheme of Chan And Ong [10]. The construction of piecewise Cubic Bézier triangular patches of Saaban [9] is slightly different that the scheme of Piah et al. [7]. Furthermore, the estimated value of the rainfall at 8 locations in peninsular Malaysia indicate that, the scheme of Saaban [9] can be used for spatial data interpolation. The method in this paper is local and guarantee to satisfy the first order of parametric continuity i.e. $C^{1}$ continuity everwhere.
TABLE I

DATA SET

\begin{tabular}{|c|c|c|c|}
\hline$i$ & $x$ & $y$ & $z$ \\
\hline 1 & 0 & 0 & 0 \\
\hline 2 & 0.2 & 0.2 & 0 \\
\hline 3 & 0.5 & 0.2 & 0 \\
\hline 4 & 0.4 & 0.4 & 0 \\
\hline 5 & 0.75 & 0.35 & 0 \\
\hline 6 & 0 & 0.5 & 1 \\
\hline 7 & 0.25 & 0.5 & 0.5 \\
\hline 8 & 0.25 & 0.75 & 1 \\
\hline 9 & 0.55 & 0.75 & 0.4 \\
\hline 10 & 0.7 & 0.6 & 0 \\
\hline 11 & 0.5 & 1 & 1 \\
\hline 12 & 0 & 1 & 1 \\
\hline 13 & 0.35 & 0 & 0 \\
\hline 14 & 0.8 & 0 & 0 \\
\hline 15 & 0.1 & 0.85 & 1 \\
\hline 16 & 0 & 0.25 & 0.5 \\
\hline 17 & 0.8 & 1 & 0.4 \\
\hline 18 & 2 & 0 & 0 \\
\hline 19 & 1.4 & 0.3 & 0.02725 \\
\hline 20 & 1.75 & 0.45 & 0 \\
\hline 21 & 1.2 & 0.45 & 0 \\
\hline 22 & 1.45 & 0.5 & 0.90451 \\
\hline 23 & 1.6 & 0.3 & 0.02725 \\
\hline 24 & 1.25 & 0.7 & 0 \\
\hline 25 & 1.4 & 0.8 & 0 \\
\hline 26 & 1.65 & 0.75 & 0 \\
\hline 27 & 2 & 1 & 0 \\
\hline 28 & 1.25 & 0 & 0 \\
\hline 29 & 1.7 & 0 & 0 \\
\hline 30 & 1.25 & 1 & 0 \\
\hline 31 & 1.7 & 1 & 0 \\
\hline 32 & 2 & 0.35 & 0 \\
\hline 33 & 2 & 0.7 & 0 \\
\hline 34 & 1.05 & 0.2 & 0 \\
\hline 35 & 1 & 0.5 & 0 \\
\hline 36 & 0.95 & 0.8 & 0 \\
\hline
\end{tabular}

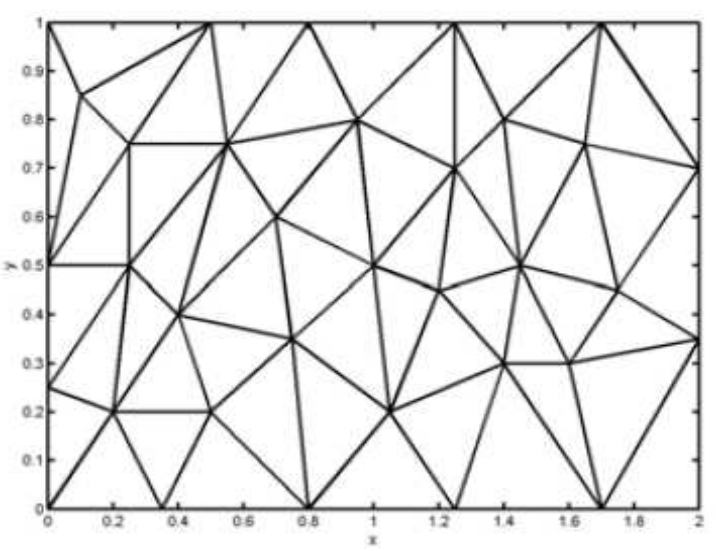

Fig. 6. Delaunay triangulation for data in Table I 


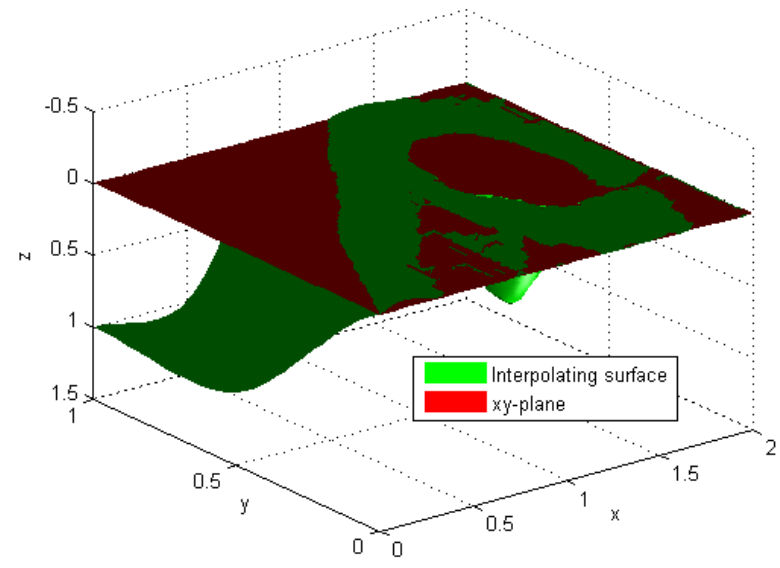

7(a) without positivity preserving

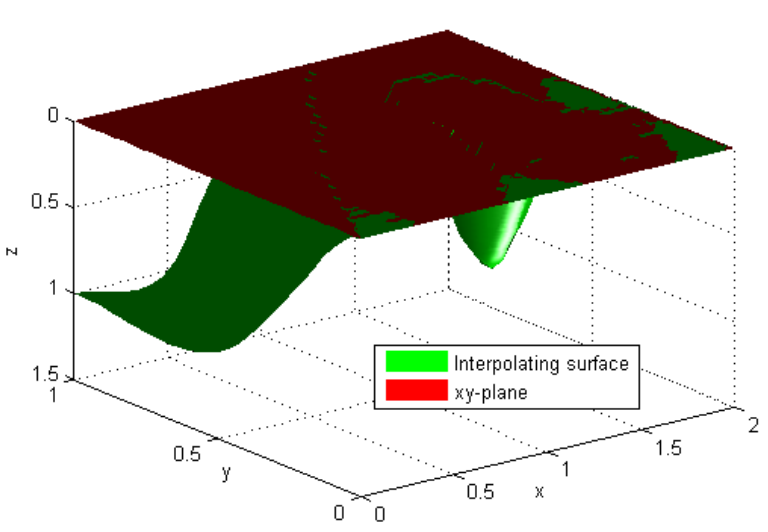

7(b) with positivity preserving

Fig. 7. Chan and Ong [2] scheme

Meanwhile the method in Skala [15] is global and involving a lot of calculation since we need to solve a large systems of linear equations that involving many unknowns. Note that, the scheme of Chang and Said [13] also can be used to produce positive interpolating scattered data surface, but the main drawback is the computation time will be increased due to involving higher degree Bézier triangular patches (degree 5). Thus the scheme of Saaban [9] is simple to be used and recommended for positivity preserving of scattered data. The following is an algorithm that can be used for the computer implementation for all three scattered data schemes discussed in this study.

Algorithm 1: Algorithm for scattered data interpolation.

Input: Data points

Output: Scattered data interpolation surfaces

Step 1: Use Delaunay triangulation to triangulate the domain of data points.

Step 2: Estimate the derivatives at the data points and then calculate Bézier ordinates values for each triangular patch Step 3: Generate the surfaces without positivity preserving conditions.
Step 4: Modify the derivatives if the Bézier ordinates does not satisfy Theorems $1-3$, respectively.

Step 5: Generate the surfaces with positivity preserving conditions.

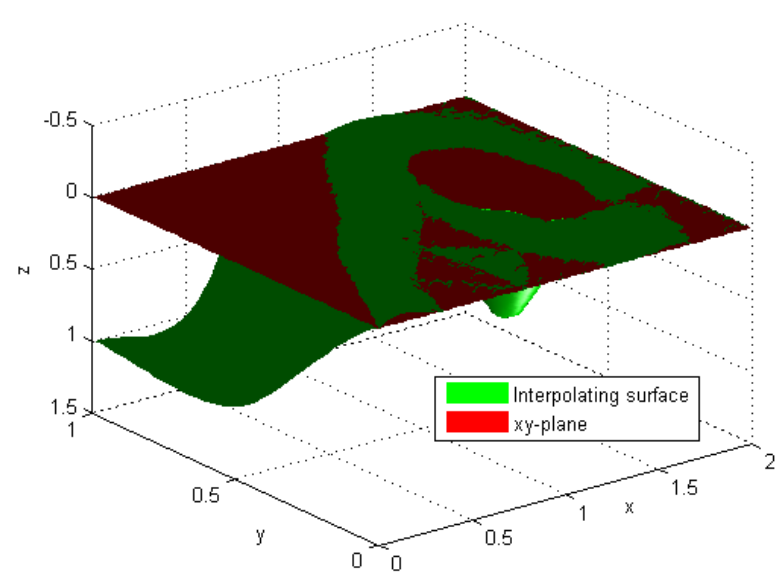

8(a) without positivity preserving

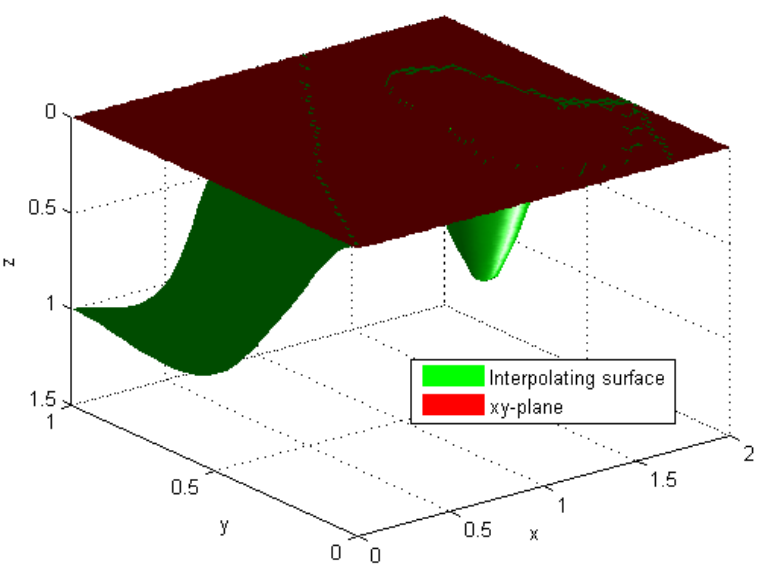

8(b) with positivity preserving

Fig. 8. Piah et al. [7] scheme

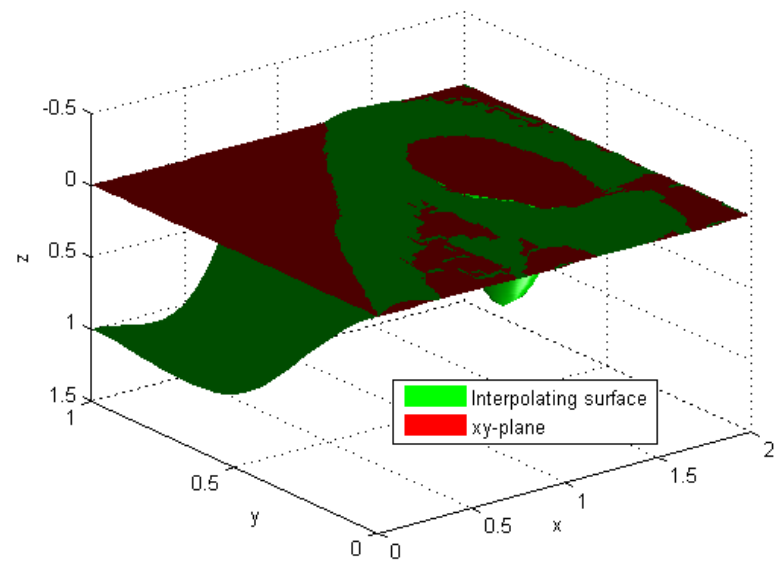

9(a) without positivity preserving 


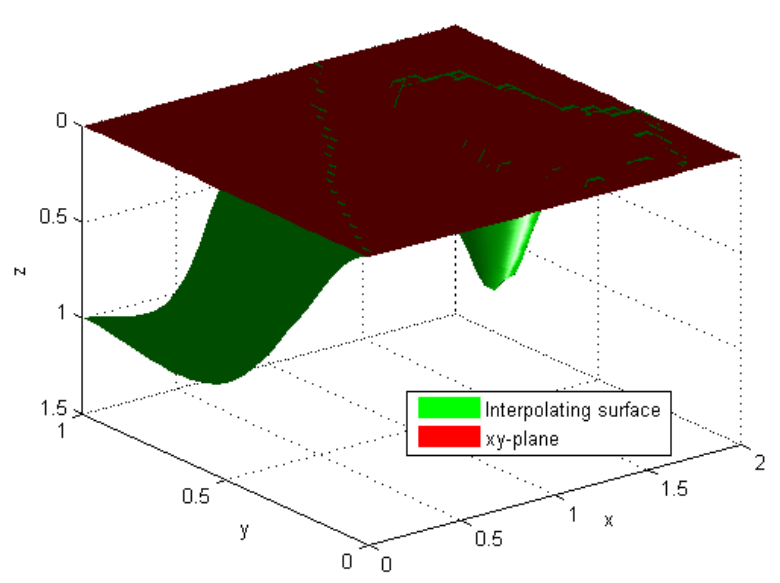

9(b) with positivity preserving

Fig. 9. Saaban [14] scheme

TABLE II

RAINFALL ESTIMATION

\begin{tabular}{|l|l|c|c|c|}
\hline & \multicolumn{1}{|c|}{ Location } & Longitude & Latitude & Rainfall (mm) \\
\hline $\mathbf{1}$ & Jitra & 100.4167 & 6.2667 & 210.16343 \\
\hline $\mathbf{2}$ & Kepala Batas & 100.4333 & 5.5167 & 186.05144 \\
\hline $\mathbf{3}$ & Kuala Kangsar & 100.9420 & 4.7667 & 267.87578 \\
\hline $\mathbf{4}$ & Genting Highland & 101.8000 & 3.4000 & 573.53521 \\
\hline $\mathbf{5}$ & Jertih & 102.5000 & 5.7500 & 290.92443 \\
\hline $\mathbf{6}$ & Nilai & 101.8000 & 2.8167 & 121.95462 \\
\hline $\mathbf{7}$ & Segamat & 102.8167 & 2.5000 & 297.15863 \\
\hline $\mathbf{8}$ & Kangar & 100.2000 & 6.4333 & 320.08024 \\
\hline
\end{tabular}

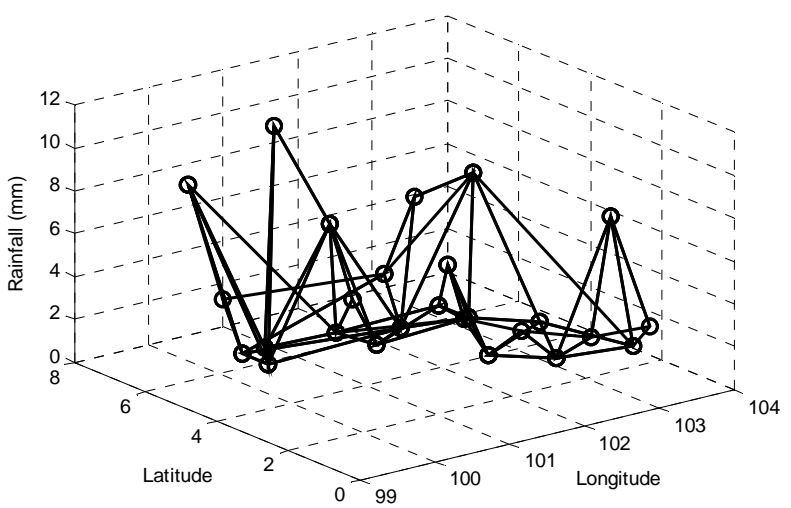

Fig. 10. Linear Interpolant for rainfall data

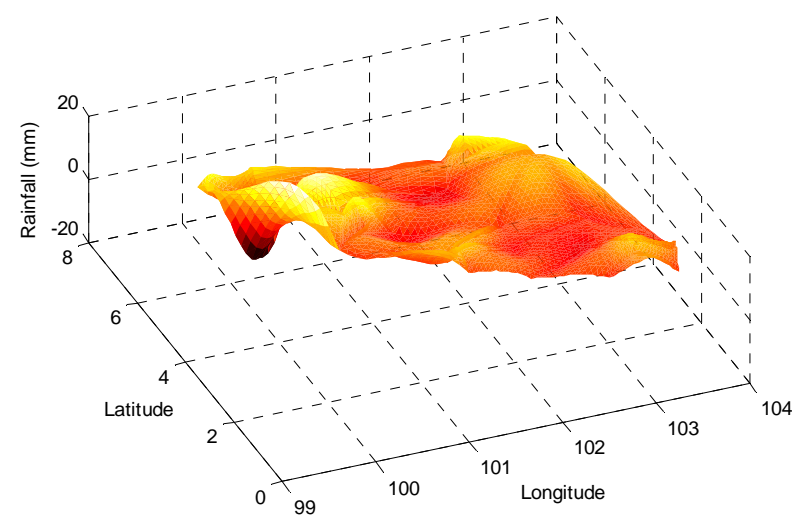

11(a) without positivity preserving

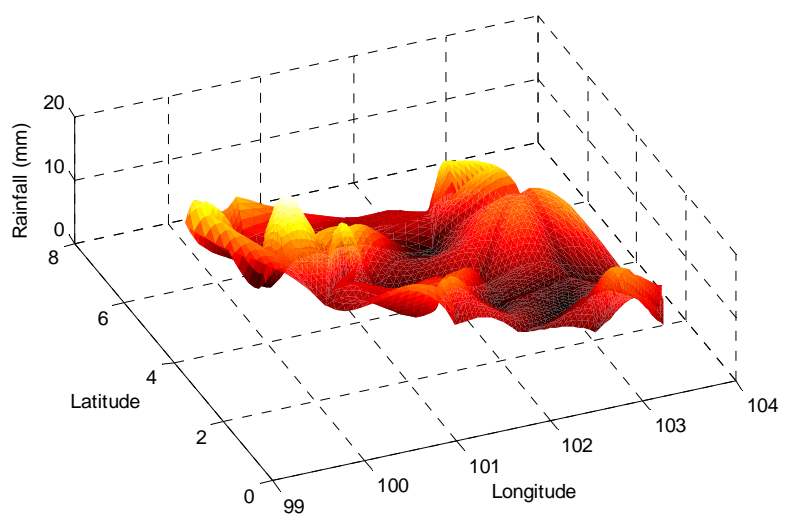

11(b) with positivity preserving

Fig. 11. Surface interpolation for rainfall data

\section{CONCLUSIONS}

This paper discusses the applications of three differences scattered data interpolation methods for positivity preserving interpolation. To examine the performance of all three methods, we execute all three methods to interpolate Data Set I given in Table I. All methods are capable to preserves the positivity of the data except for Chang and Ong [10] scheme, where there some parts on the interpolating surfaces that lie below xy-plane- as clearly shown in Fig. 7. The other two schemes i.e. Piah et al. [8] and Saaban [9] was perfectly simulate non-negative values. Since Saaban [9] interpolate Data Set I very well, we use the scheme of Saaban [9] to estimate the unknown value of rainfall at certain location. A MATLAB programming has been written in order to cater this spatial interpolation problem. The users need to input the longitude and latitude of the desired location. The estimated value was calculated by using the cubic Bézier triangular patches by applying Theorem 3. From Fig. 11, we conclude that Saaban [9] scheme can be used to estimate the unknown value of the rainfall at the desired location. The rainfall estimated data is given in Table II. Fig. 11(b) clearly shows that Saaban [9] scheme give positive values for all simulated locations.

In our next research, we intend to apply the scheme of [9] to predict rainfall volume or amount as well as do the comparison with its actual rainfall value. This will be usefull for the relevant agency in predicting the rainfall and prepare the appropriate action plan when the heavy rain is happened. 
Future works will be applying the scheme for geophysical and meteorological data sets such as seismic and earthquake intensity. This can be achieved by considering the repective information i.e. starting with triangulating all $2 \mathrm{D}$ data then employ the scattered data scheme to produce the required surface for spatial interpolation. Starting point will be [21] and [22].

\section{ACKNOWLEDGMENT}

This research is fully supported by Universiti Teknologi PETRONAS (UTP) through a research grants: STIRF 0153AA-D91 and YUTP: 0153AA-H24 (Spline Triangulation for Spatial Interpolation of Geophysical Data). Much of the work were carried out while the first author is visiting School of Mathematical Sciences, UKM and Faculty of Information Science and Technology, UKM on $19^{\text {th }}$ February 2018 until $22^{\text {nd }}$ February 2018.

\section{REFERENCES}

[1] S.K., Lodha, and R., Franke, "Scattered Data Techniques for Surfaces,' in Scientific Visualization," in Dagsthul 97 Proceedings, IEEE Computer Society Press, 2000, pp. 189-230.

[2] R. Franke, and G.M. Nielson, "Scattered data interpolation of large Sets of scattered data," Intl. J. Numerical Methods in Eng., vol. 15, pp. 1691-1704, 1980.

[3] R. Franke, "Scattered data interpolation: tests of some methods," Math. Comput., vol. 38, pp. 181-200, 1982.

[4] R. Franke, and G.M. Nielson, Scattered data interpolation and applications: a tutorial and survey, In H. Hagen and D. Roller Ed. Geometric Modelling: Methods and Applications, Berlin: Springer, pp. 131-160, 1991.

[5] W., Li, Y. Y. Han, and J. X. Chen, "Triangular-patch based texture synthesis over arbitrary surfaces,", Proceedings of 2008 Congress on Image and Signal Processing, pp. 441-445, 2008.

[6] L., Miroslav, S., Zbynek, and V. Jan, "Smooth surface interpolation using patches with rational offsets," Computer Aided Geomectric Design, Preprint, Feb 2018.

[7] A.R.Mt., Piah, T.N.T., Goodman, and K., Unsworth, "Positivitypreserving scattered data interpolation," Lecture Notes in Computer Sciences (LNCS) 3604, pp. 336-349, 2005
[8] A., Saaban, A.A., Majid, and A.R.Mt., Piah, "Visualization of Rainfall Data Distribution Using Quintic Triangular Bézier Patches," Bulletin of Malaysian Mathematical Sciences Society, vol. 32(2), pp. 137-150, 2009.

[9] A., Saaban. "Parametric Interpolation to Scattered Data," $\mathrm{PhD}$ thesis, Universiti Sains Malaysia, Penang, Malaysia, 2008.

[10] E.S., Chan and B.H., Ong., "Range restricted scattered data interpolation using convex combination of cubic Bézier triangles," Journal of Computational and Applied Mathematics, vol. 136, pp. $135-147,2001$

[11] T.N.T., Goodman, and H.B., Said, "A $C^{1}$ - triangular interpolation suitable for scattered data interpolation," Communications in Applied Numerical Methods, vol. 7, pp. 479-485, 1991.

[12] T.A. Foley, and K. Optiz, Hybrid cubic Bézier triangle patches. In T. Lyche and L.L. Schumaker (eds.), Mathematical Methods in Computer Aided Geometric Design II, Academic Press, 275-286, 1992.

[13] L. H. T. Chang, and H. B. Said, "A $C^{2}$ triangular patch for the interpolation of functional scattered data," Computer Aided Design vol. 29(6), pp. 407 - 412, 1997.

[14] K.W., Brodlie, M.R., Asim and K., Unsworth, "Constrained visualization using Shepard interpolation family," Computers and Graphics Forum, vol. 24(4), pp. 809-820, 2005.

[15] V. Skala, "RBF Interpolation with CSRBF of Large Data Sets", Procedia of Computer Science, Volume108, , pp. 2433-2437, 2017

[16] G. Farin, "Triangular Bernstein-Bézier patches," Computer Aided Geometric Design, vol.3, pp. 83-127, 1986.

[17] G. Farin, Curves and Surfaces for CAGDesign: A Practicle Guide, 5th ed., C. Palmer, Ed. , San Diego, USA: Morgan Kaufmann, 2001.

[18] https://www.quora.com/What-is-a-polygon-in-gaming.

[19] https://stackoverflow.com/questions/31191790/b\%C3 \%A9ziertriangles-and-n-patches

[20] http://www.wamit.com/manualv7.2/wamit_v72manualch10.html.

[21] Thota Sivasankar, Dheeraj Kumar, Hari Shanker Srivastava, Parul Patel, Advances in Radar Remote Sensing of Agricultural Crops: A Review, International Journal on Advanced Science, Engineering and Information Technology, Vol. $8 \quad$ (2018) No. 4, DOI:10.18517/ijaseit.8.4.5797

[22] Farida -, Nifatamah Makaje, Phattrawan Tongkumchum, Aniruth Phon-On, Jetsada Laipaporn, Natural Cubic Spline Model for Estimating Volatility, International Journal on Advanced Science, Engineering and Information Technology, Vol. 8 (2018) No. 4, DOI:10.18517/ijaseit.8.4.3107 